\title{
O ensino de Estrutura de Dados auxiliado por uma Plataforma Didática na Web
}

\author{
Lucas Fernando Borges ${ }^{1}$, Ana Cláudia Martinez ${ }^{1}$, Thiago Pirola Ribeiro \\ ${ }^{1}$ Faculdade de Computação - Universidade Federal de Uberlândia (UFU) \\ - Uberlândia - MG - Brazil \\ \{lucas.fernando, anacmartinez, tpribeiro\}@ufu.br
}

\begin{abstract}
The initial academic disciplines of programming and algorithms in Computer Science courses require an abstraction level that was not necessary in high school. Based on previous studies of the greatest difficulties of students in courses of programming and trying to collaborate to reduce such difficulties, the present work provides a simulator, developed in a Web platform, which permits students to manipulate data structures with visual results.
\end{abstract}

Resumo. As disciplinas iniciais de programação e algoritmos dos cursos da área de computação exigem um certo grau de abstração que, normalmente, não é cobrado no ensino médio. A partir de estudos prévios das maiores dificuldades de alunos dos cursos de programação e, tentando colaborar para diminuir as barreiras iniciais, este trabalho apresenta um simulador desenvolvido em plataforma Web, no qual é possível realizar manipulações de estruturas de dados com resultados visuais.

\section{Introdução}

Um grande desafio no ensino atual é conseguir utilizar a tecnologia disponível no dia a dia como um instrumento para tornar os conteúdos mais atrativos, compreensíveis e motivadores para os estudantes que, diariamente, estão altamente tecnológicos e conectados.

Nesse intuito, o uso de simuladores auxilia os estudantes na visualização do conteúdo abordado, além de permitir interação com o objeto de estudo, permitindo um melhor desenvolvimento do conteúdo e também a melhora das habilidades de aprendizado e investigação.

$\mathrm{Na}$ literatura encontram-se diversos experimentos realizados com o intuito de comprovar a eficácia do uso de simuladores no ensino em diversas áreas. [Uzezi and Deya 2020] utilizaram simuladores para auxiliar os alunos no estudo de química nas reações ácido-base na Taraba State University e puderam comprovar a melhora no desempenho dos estudantes. [Martins et al. 2020] utilizaram um simulador para aulas de química, porém para o ensino médio de uma escola pública em Irituia-PA e constataram que os estudantes tiveram um melhor entendimento da matéria e maior assiduidade nas aulas.

$\mathrm{Na}$ área da computação, do mesmo modo como em outras áreas, as disciplinas podem utilizar simuladores para auxiliar no entendimento de conceitos que necessitam ser abstraídos ou até mesmo montar e/ou executar um estrutura que fisicamente teria um custo alto. [Martins 2016] utilizou o simulador Cisco Packet Tracer da Cisco conseguindo melhorar a percepção cognitiva dos alunos, contribuindo para o aprendizado e 
[Ribeiro et al. 2014] desenvolveram um simulador para rotinas especificas para a disciplina de Sistemas Operacionais.

As disciplinas que envolvem programação e estrutura de dados são consideradas desafiadoras para muitos alunos por não conseguirem abstrair os desafios propostos, resultando em altos índices de reprovação e, em última instância, no abandono do curso. Esse problema não é novo, sendo que em 2006 [dos Santos and Costa 2006] já analisavam metodologias e ambientes de ensino para Algoritmos e Estruturas de Dados, baseadas nas ferramentas computacionais: ASTRAL, EDDL, TED, DADS e TBC-AED. Além dessas ferramentas, existem diversos simuladores para conteúdos mais complexos de estruturas de dados, porém com o foco em estruturas básicas (vetor, matriz, ponteiros, filas, pilhas e listas), quase nada é encontrado.

Na Tabela 1 foram analisados 4 simuladores, porém não contemplam todos os itens básicos, sendo o Data Structure Visualizations um dos que mais possuem algoritmos simulados, sendo este um dos mais citados na literatura.

Tabela 1. Resumo das opções disponibilizadas pelos simuladores analisados.

\begin{tabular}{lccccccc}
\hline \multicolumn{1}{c}{ Simuladores } & Vetor & Matriz & Pilha & Fila & Lista & Árvore & Ordenação \\
\hline $\begin{array}{l}\text { Data Structure Visuali- } \\
\text { zations [Galles 2011] }\end{array}$ & $\mathrm{X}$ & & $\mathrm{X}$ & $\mathrm{X}$ & $\mathrm{X}$ & $\mathrm{X}$ & $\mathrm{X}$ \\
$\begin{array}{l}\text { VisuAlgo } \\
\text { [Halim et al. 2012] }\end{array}$ & $\mathrm{X}$ & & & $\mathrm{X}$ & $\mathrm{X}$ & $\mathrm{X}$ \\
$\begin{array}{l}\text { Data Structure Simula- } \\
\text { tion [Bembalkar 2018] }\end{array}$ & & $\mathrm{X}$ & & & $\mathrm{X}$ & \\
$\begin{array}{l}\text { Data structure and } \\
\text { algorithm tutorial } \\
\text { [ZeroZiano 2019] }\end{array}$ & & & & $\mathrm{X}$ & $\mathrm{X}$ & $\mathrm{X}$ \\
\hline
\end{tabular}

Nas disciplinas de Programação e de Estrutura de Dados, os professores desenham no quadro para tentar explicar o funcionamento de certas estruturas. Isso, por muitas vezes, acaba por desestimular os alunos na tentativa de abstração do conteúdo explicado, pois os alunos podem se sentirem constrangidos em tentarem interagir com o professor, pedindo que o mesmo crie novos exemplos "do jeito" que eles estão pensando.

O presente trabalho propõe o desenvolvimento de um simulador para vetor, matriz, ponteiro, pilha e fila, tendo como diferencial a proposta de unir a visualização das estruturas com a codificação e a forma como as ações ocorrem na memória simulada.

\section{Metodologia}

Para esse trabalho foi estabelecida uma abordagem metodológica baseada no grande número de reprovações ocorridas nas matérias de programação dos cursos de Computação, mais especificamente em matérias iniciais de Programação de Computadores e Estruturas de Dados.

Realizou-se uma pesquisa para identificar o percentual de reprovações nas matérias relacionadas a programação, especificamente no curso de Bacharelado em Sistemas de Informação oferecido pela Universidade Federal de Uberlândia no campus Monte 
Carmelo. Percebeu-se que as técnicas de ensino aplicadas baseavam-se nas bibliografias e métodos clássicos e um índice de reprovação superior a 60\%, evidenciando a necessidade da utilização de novas abordagens.

Buscou-se por simuladores que apresentassem abordagens de estruturas específicas de programação, porém os softwares encontrados são incompletos e não abordam todas as estruturas básicas. Com isso, desenvolveu-se um esboço com as estruturas e telas para um simulador unindo a linguagem de programação, o conceito e o que ocorre na memória como efeito do comando executado.

\section{Resultados}

O presente trabalho desenvolveu o simulador DebugandoED ${ }^{1}$ que, diferentemente dos simuladores analisados, realiza a simulação de vetor, matriz, ponteiros, pilha e fila. Além de demostrar a estrutura visual de cada conteúdo, aborda como seria o código em linguagem $\mathrm{C}$ de cada operação de manipulação das referidas estruturas e a forma como as ações ocorrem em uma memória simulada.

A Figura 1 ilustra uma das estruturas criadas que de maneira geral, o usuário define o tamanho, e posteriormente o sistema criará a estrutura com o tamanho escolhido. Em (A) são exibidas todas as opções de manipulação, contendo inserção e remoção de conteúdo, exibição de todos os valores inseridos, como também a opção de mostrar a posição em que se encontra o início (no caso da fila). Cada operação realizada pelo usuário, como inserir e remover valores, gera um código de programação ${ }^{2}$ referente a essa operação (B). (C) ilustra a memória simulada e, nesse caso, todas as variáveis usadas são mostradas nesse espaço. São apresentadas três colunas: a primeira ilustra as variáveis em si, a segunda ilustra o endereço de memória simulado e a última o conteúdo inserido ou lixo de memória. Automaticamente todas as operações realizadas no simulador afetam as informações na memória, assim a cada interação essas são atualizadas. (D) ilustra a forma visual da ação executada.

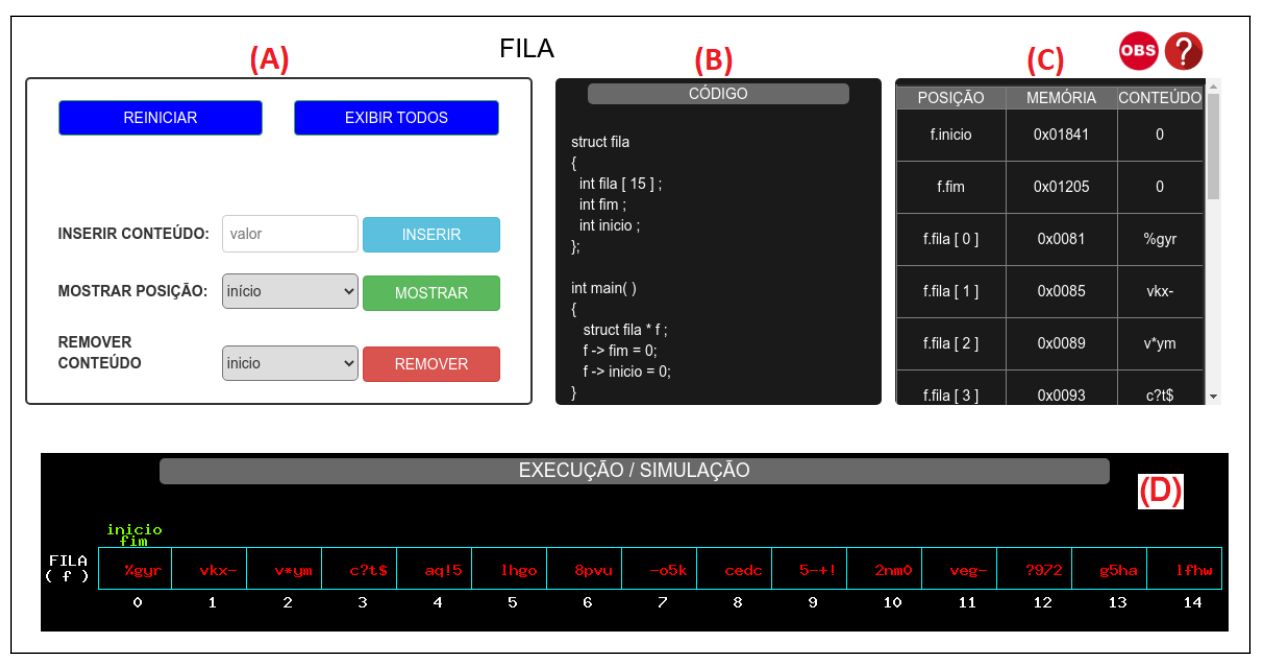

Figura 1. Tela exemplificando o simulador desenvolvido.

\footnotetext{
${ }^{1} \mathrm{O}$ simulador proposto pode ser acessado em: https : / / debugandoed. facom. ufu.br

${ }^{2} \mathrm{~A}$ linguagem $\mathrm{C}$ foi escolhida por ser a utilizada em sala de aula.
} 


\section{Conclusão}

Com a pandemia do COVID-19, a plataforma está sendo utilizada em um dos cursos, sem a avaliação do aprendizado dos alunos, apenas para que os professores e os alunos deem feedbacks anônimos sobre a utilização e as sugestões de possíveis melhorias.

Após a aprovação no Comitê de Ética, serão realizados experimentos em turmas de várias universidades e departamentos diferentes, com a separação de percentual que utilizará e outro que não utilizará a plataforma para a validação do auxílio gerado pelo uso. Espera-se que os alunos que utilizarem o simulador proposto possam assimilar melhor o conteúdo e possivelmente demonstrarem maior motivação para os estudos.

Já estão em fase de implementação as estruturas: listas, árvores e ordenação, porém ainda não estão disponibilizadas/testadas. Além disso, o simulador contará com versões em português e inglês.

\section{Referências}

Bembalkar, A. (2018). Data structure simulation. https://play.google. com/store/apps/details?id=com.datastructure.akshay . datastructuresimulation\&hl=pt_BR. Acessado em 18 de março de 2021.

dos Santos, R. P. and Costa, H. A. X. (2006). Análise de metodologias e ambientes de ensino para algoritmos, estruturas de dados e programação aos iniciantes em computação e informática. INFOCOMP Journal of Computer Science, 5(1):41-50.

Galles, D. (2011). Data structure visualizations. https://www.cs.usfca.edu/ qalles/visualization/. Acessado em 15 de março de 2021.

Halim, S., Koh, Z. C., Loh, V. B. H., and Halim, F. (2012). Learning algorithms with unified and interactive web-based visualization. Olympiads in Informatics, 6.

Martins, A. M. d. C. (2016). O uso de simuladores no ensino de redes: um estudo de caso no ensino profissional. Master's thesis, Universidade Católica Portuguesa.

Martins, S. O., Serrão, C. R. G., Silva, M. D. D. B., and dos Reis, A. S. (2020). O uso de simuladores virtuais na educação básica: Uma estratégia para facilitar a aprendizagem nas aulas de química. Revista Ciências \& Ideias ISSN: 2176-1477, 11(1):216-233.

Ribeiro, T. P., Lima, R. L. B., and Lobo, E. A. (2014). Simuladores de gerência de memória e processador para auxílio às aulas teóricas de sistemas operacionais. In Brazilian Symposium on Computers in Education (Simpósio Brasileiro de Informática na Educação-SBIE), volume 25, page 1028.

Uzezi, J. G. and Deya, G. D. (2020). Effect of computer simulation on secondary school students' academic achievement in acid-base reactions. ATBU Journal of Science, Technology and Education, 8(1):286-295.

ZeroZiano (2019). Data structure and algorithm tutorial. https://play.google. $\mathrm{com} / \mathrm{store} / \mathrm{apps} /$ details? id=com.zero.dsa. Acessado em 18 de março de 2021 . 